\title{
Membrane Resonance in Bursting Pacemaker Neurons of an Oscillatory Network Is Correlated with Network Frequency
}

\author{
Vahid Tohidi ${ }^{1}$ and Farzan Nadim ${ }^{1,2}$ \\ ${ }^{1}$ Federated Department of Biological Sciences, Rutgers University, Newark, New Jersey 07102, and 2Department of Mathematical Sciences, New Jersey \\ Institute of Technology, Newark, New Jersey 07102
}

\begin{abstract}
Network oscillations typically span a limited range of frequency. In pacemaker-driven networks, including many central pattern generators (CPGs), this frequency range is determined by the properties of bursting pacemaker neurons and their synaptic connections; thus, factors that affect the burst frequency of pacemaker neurons should play a role in determining the network frequency. We examine the role of membrane resonance of pacemaker neurons on the network frequency in the crab pyloric CPG. The pyloric oscillations (frequency of $\sim 1 \mathrm{~Hz}$ ) are generated by a group of pacemaker neurons: the anterior burster (AB) and the pyloric dilator (PD). We examine the impedance profiles of the $\mathrm{AB}$ and $\mathrm{PD}$ neurons in response to sinusoidal current injections with varying frequency and find that both neuron types exhibit membrane resonance, i.e., demonstrate maximal impedance at a given preferred frequency. The membrane resonance frequencies of the $\mathrm{AB}$ and $\mathrm{PD}$ neurons fall within the range of the pyloric network oscillation frequency. Experiments with pharmacological blockers and computational modeling show that both calcium currents $I_{\mathrm{Ca}}$ and the hyperpolarization-activated inward current $I_{h}$ are important in producing the membrane resonance in these neurons. We then demonstrate that both the membrane resonance frequency of the PD neuron and its suprathreshold bursting frequency can be shifted in the same direction by either direct current injection or by using the dynamic-clamp technique to inject artificial conductances for $I_{h}$ or $I_{\mathrm{Ca}}$. Together, these results suggest that membrane resonance of pacemaker neurons can be strongly correlated with the CPG oscillation frequency.
\end{abstract}

\section{Introduction}

A variety of neurons in different systems exhibit subthreshold membrane potential resonance, a maximum voltage response to low-amplitude subthreshold oscillatory input currents at a certain preferred (resonant) frequency (Gimbarzevsky et al., 1984; Art et al., 1986; Llinás and Yarom, 1986; Puil et al., 1986; Hutcheon et al., 1994). Membrane resonance has been associated with low-amplitude subthreshold oscillations of membrane potential (Llinás, 1988; Gutfreund et al., 1995; Lampl and Yarom, 1997; Leung and $\mathrm{Yu}, 1998$ ), which, in turn, correlates with the probability of action potential generation (Engel et al., 2008). Although the relevance of membrane resonance for field oscillations is relatively well known, few studies have explored a direct relationship between membrane resonance and suprathreshold neuronal activity (but see Brunel et al., 2003; Richardson et al., 2003; Engel et al., 2008). Additionally, membrane resonance has been studied primarily in tonically firing neurons that lack intrinsic bursting properties, and thus much less is known about the relevance of membrane resonance in bursting neurons (D'Angelo et al., 2001; Izhikevich et al., 2003).

Central pattern generator (CPG) networks often involve bursting neurons whose stable oscillatory activity is the basis of rhythmic motor behavior. In many CPGs, oscillatory activity

Received Jan. 2, 2009; revised March 15, 2009; accepted April 2, 2009

This work was supported by National Institutes of Health Grant MH-60605. We thank Jorge Golowasch and Hua-an Tseng for the useful discussions and comments on this manuscript.

Correspondence should be addressed to Farzan Nadim, Department of Mathematical Sciences, New Jersey Institute of Technology, 323 Martin Luther King Boulevard, Newark, NJ 07102. E-mail: farzan@njit.edu.

D0I:10.1523/JNEUROSCI.0545-09.2009

Copyright $\odot 2009$ Society for Neuroscience $\quad$ 0270-6474/09/296427-09\$15.00/0 arises from the coordinated firing of (conditional) pacemaker neurons that produce bursting activity (Moulins and Cournil, 1982; Hooper and Marder, 1984; Bellingham, 1998). Because bursting oscillations are often associated with low-threshold activated ionic currents, it is possible that these currents may give rise to membrane resonance. If so, the membrane potential resonance properties of these bursting neurons might be correlated with their suprathreshold bursting activity and thus contribute to network level oscillations in CPGs.

We examine the role of membrane resonance in the crustacean pyloric network, a well studied CPG that is characterized by very stable oscillations (frequency of $\sim 1 \mathrm{~Hz}$ ) produced by a group of pacemaker neurons. This pacemaker group involves two neuron types, the anterior burster $(\mathrm{AB})$ and the pyloric dilator $(\mathrm{PD})$, that are strongly electrically coupled and produce synchronized bursting activity. We demonstrate that the pyloric pacemaker neurons exhibit membrane resonance at a frequency that falls in the range of the network oscillation frequencies. We then explore the ionic mechanisms that underlie the resonance properties of the pacemaker neurons. Finally, we demonstrate that the frequency of membrane resonance of the pacemaker neurons can be correlated with their suprathreshold bursting frequency and therefore the network oscillation frequency. These results suggest that membrane resonance properties of pacemaker neurons can be an important factor in determining the frequency of rhythmic activity in CPGs.

\section{Materials and Methods}

Experimental protocols. Experiments were performed on adult male crabs (Cancer borealis) purchased from local distributors. The animals were 
Table 1. Parameters of dynamic clamp-injected currents

\begin{tabular}{|c|c|c|c|c|c|c|c|c|c|c|c|}
\hline Unit & $g_{\max }(\mathrm{nS})$ & $E_{\mathrm{rev}}(\mathrm{nS})$ & $V_{m}(\mathrm{mV})$ & $k_{m}(\mathrm{~ms})$ & $\tau_{m h i}(\mathrm{~ms})$ & $\tau_{m / o}(\mathrm{~ms})$ & $p$ & $V_{h}(\mathrm{mV})$ & $k_{h}(\mathrm{mV})$ & $\tau_{h h i}(\mathrm{~ms})$ & $\tau_{\text {hlo }}(\mathrm{ms})$ \\
\hline$I_{C a, d y n}$ & $5,-5$ & 110 & -30 & -20 & 400 & 25 & 3 & & & & \\
\hline$I_{h, d y n}$ & $12,-12$ & -20 & -50 & 6 & 250 & 50 & 1 & & & & \\
\hline
\end{tabular}

Note that the positive (negative) $g_{\max }$ values indicated were the maximum values used to shift the resonance peak to higher (lower) frequencies.

maintained in artificial seawater tanks at $12-15^{\circ} \mathrm{C}$ until use. They were anesthetized by cooling on ice for 15-30 min before dissection. The stomatogastric nervous system [including the stomatogastric (STG), esophageal, and paired commissural ganglia] was removed using standard methods (Selverston et al., 1976; Harris-Warrick et al., 1992) and pinned down in a Sylgard-coated Petri dish. The STG was desheathed to expose the cell bodies to intracellular electrodes and to effective superfusion of the neurons using normal saline at $11-13^{\circ} \mathrm{C}, \mathrm{pH} 7.4$, containing the following (in mM): $11 \mathrm{KCl}, 440 \mathrm{NaCl}, 13 \mathrm{CaCl}_{2} \cdot 2 \mathrm{H}_{2} \mathrm{O}, 26$ $\mathrm{MgCl}_{2} \cdot 6 \mathrm{H}_{2} \mathrm{O}, 11.2$ Trizma base, 5.1 and maleic acid.

Microelectrodes for neuron impalement were pulled using a FlamingBrown micropipette puller (P97; Sutter Instruments) and filled with 0.6 $\mathrm{M} \mathrm{K}_{2} \mathrm{SO}_{4}$ plus $0.02 \mathrm{M} \mathrm{KCL}$ (resistance of $8-12 \mathrm{M} \Omega$ for current injection, $15-25 \mathrm{M} \Omega$ for recording). Neuron identification was accomplished by matching intracellular action potential recordings to extracellular recordings from identified nerves (Selverston et al., 1976; Harris-Warrick et al., 1992). Intracellular somatic impalements were done with two sharp microelectrodes simultaneously (one for current injection and the other one for recording of voltage response) using Axoclamp 2B amplifiers (Molecular Devices), and extracellular recordings were filtered (bandpass $100-5000 \mathrm{~Hz}$ ) and amplified using a differential alternating current amplifier model 1700 (A-M Systems).

In experiments measuring the resonance property of neurons, the ganglion was superfused with $0.1 \mu \mathrm{M}$ tetrodotoxin (TTX) (Biotium). We used 5-10 $\mathrm{mM} \mathrm{Cs}^{+}$for blocking $I_{h}$ and $12.9 \mathrm{mM} \mathrm{Mn}^{2+}$ plus $0.1 \mathrm{~mm} \mathrm{Ca}^{2+}$ for blocking $I_{\mathrm{Ca}}$ (Golowasch and Marder, 1992). Although ZD7288 (4ethylphenylamino-1,2-dimethyl-6-methylaminopyrimidinium chloride) is as effective for blocking $I_{h}$, it was not used because $\mathrm{Cs}^{+}$is a cleaner blocker in the stomatogastric nervous system and its effect is rapidly reversed (Peck et al., 2006). We used 10 mm tetraethylammonium (TEA) (Sigma-Aldrich) and $30 \mu \mathrm{M}$ Riluzole (Sigma-Aldrich) for blocking potassium currents and persistent sodium current, respectively. Photoinactivation (Miller and Selverston, 1979) of AB neurons was performed by microinjecting them with Alexa Fluor 568 hydrazide and sodium salt in $200 \mathrm{~mm} \mathrm{KCl}$ (Invitrogen) and illuminating the STG with a green laser.

Impedance and ZAP current. The ZAP function is a sinusoidal function with fixed amplitude but sweeping frequencies in a given range. The ZAP current can be described as follows:

$$
I_{\mathrm{ZAP}}=I_{\max } \sin (2 \pi f(t) \times t),
$$

where $f(t)$ produces the range of frequencies that the ZAP function sweeps. In our application, we used an exponential chirp function for $f(t)$ to increase the sampling duration of lower frequencies:

$$
f(t)=\frac{f_{\min }}{L}\left(e^{L t}-1\right),
$$

where

$$
L=\log _{\frac{\max }{f_{\min }}} .
$$

The amplitude of the ZAP current was selected as the minimum amplitude that produced a voltage response within the range of the ongoing PD neuron slow-wave oscillation (approximately -60 to $-33 \mathrm{mV}$ ). This amplitude was typically set to $1.5-2 \mathrm{nA}$. In some experiments (when the amplitude was $1.5 \mathrm{nA}$ ), a bias direct current (DC) pulse of $<0.25 \mathrm{nA}$ was added to the ZAP current to bring the minimum voltage (at lowest frequencies) to $-60 \mathrm{mV}$. We used $\mathrm{ZAP}$ current injection to measure the impedance of neurons as a function of the frequency of the injected ZAP current $(I)$. Impedance contains information about both amplitude and phase of oscillation and is calculated as a function of the input frequency as follows:

$$
Z(f)=\frac{\tilde{V}(f)}{\tilde{I}(f)},
$$

where $\tilde{V}$ and $\tilde{I}$ are, respectively, the Fourier transforms of the membrane potential $V$ and the ZAP current $I$ (Hutcheon and Yarom, 2000). $Z(f)$ is a complex number; the absolute value of $Z$ is the impedance power and is plotted as a function of frequency. In our results, we refer to the impedance power as impedance.

Dynamic clamp. The dynamic-clamp technique is used to inject a prescribed artificial conductance corresponding to a specific ionic current into a biological neuron (Goaillard and Marder, 2006). This specific conductance can represent an intrinsic or synaptic current. The dynamic-clamp current used was calculated as follows:

$$
I_{x}=\bar{g}_{x} m_{x}^{p} h_{x}^{q}\left(V-E_{x}\right),
$$

where $V$ is the membrane potential of the neuron recorded in real time, $x$ is the modeled ionic current, $m_{x}$ is the activation gate, $h_{x}$ is the inactivation gate, $\bar{g}_{x}$ is the maximum conductance, $E_{x}$ is the reversal potential, and $p$ and $q$ are non-negative integers. The activation and inactivation terms are sigmoidal functions of $V$ :

$$
\begin{gathered}
\frac{d x}{d t}=\frac{1}{\tau_{x}}\left(x_{\infty}(V)-x\right), \\
x_{\infty}(V)=\frac{1}{1+\exp \left(\left(V-V_{x}\right) / k_{x}\right)}, \\
\tau_{x}=\tau_{x, h i}+\left(\tau_{x, l o}-\tau_{x, h i}\right) /\left(1+\exp \left(\left(V-V_{x}\right) /\left|k_{x}\right|\right)\right),
\end{gathered}
$$

where $x=m$ or $h$. Note that the same parameters define the midpoint $\left(V_{x}\right)$ or slope $\left(k_{x}\right)$ of the sigmoidal functions defining $x_{\infty}$ and $\tau_{x}$. The sign of $k_{x}$ determines whether the sigmoidal function is increasing or decreasing as a function of $V$; thus, the definition of $x$ uses the absolute value of $k$ to ensure that $\tau_{x}=\tau_{x, l o}$ when $V \ll V_{x}$ and $\tau_{x}=\tau_{x, h i}$ when $V \gg V_{x}$.

The parameters of the currents, $I_{\mathrm{Ca} \text {,dyn }}$ and $I_{h \text {,dyn }}$, used in our dynamicclamp experiments are given in Table 1 . When simultaneous dynamicclamp current and constant-current injections were required, a Brownlee Precision Amplifier was used to add the currents before input to the Axoclamp amplifier.

Data analysis. Data analysis and measurement of impedance profiles were performed with scripts written in Matlab (version 6.5; MathWorks). The NI PCI-6070-E board (National Instruments) was used for data acquisition and for ZAP current injection with the data acquisition software Scope (version 7.71) and DClamp (version 1.54). A Digidata 1332A board was used for simultaneous data acquisition with the Clampex software. The acquired data were saved as binary files and were analyzed with Readscope (version 7.70) and Clampfit. The Clampex and Clampfit software are part of the pClamp package (pClamp 9.2; Molecular Devices). The Scope, Readscope, and Dynamic Clamp software are developed in the Nadim laboratory and are available freely at (http://stg.rutgers.edu/software/).

Computational simulations. We used the software Network (version 2.07; http://stg.rutgers.edu/software/) for the simulation of the biophysical model of the PD neuron. This model was based on the twocompartment model of the pacemaker neurons developed by SotoTreviño et al. (2005). A detailed description of the model and its parameters have been given previously (Soto-Trevino et al., 2005). We modified some parameters so that the membrane resonance frequency 
A1
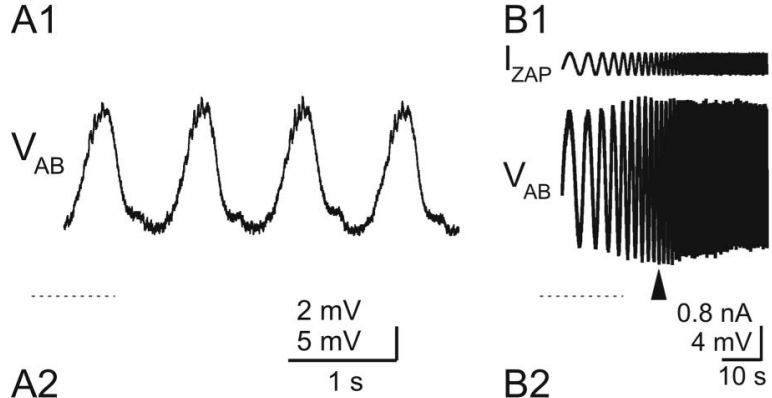

A2

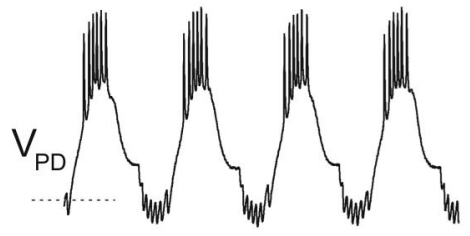

B2

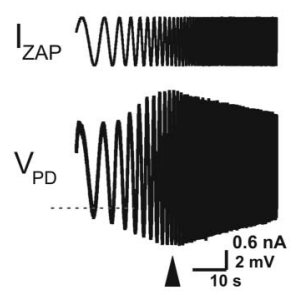

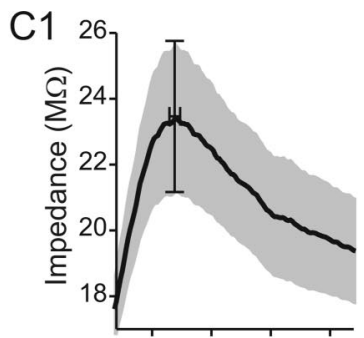

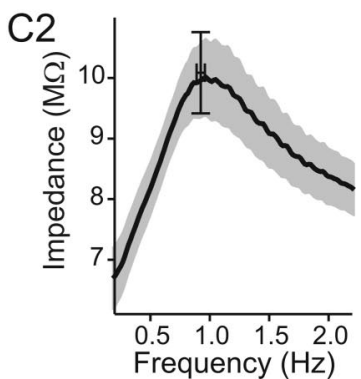

Figure 1. Membrane resonance measurement of the pyloric pacemaker neurons $A B$ and $P D$. Simultaneous recordings of the $A B$ (A1) and PD (A2) neuron oscillations during the ongoing pyloric rhythm. B1,. The voltage response of the AB neuron to a $60 \mathrm{~s} Z A P$ current injection (top trace) that sweeps a frequency range between 0.2 and $3 \mathrm{~Hz}$ indicates a maximum amplitude at a preferred resonance frequency ( $f_{\max }$ indicated by arrowhead). B2, The voltage trace of the PD neuron in response to a similar ZAP current also shows a resonance frequency $f_{\max }$ (arrowhead). The small horizontal dashed lines in $\boldsymbol{A}$ and $\boldsymbol{B}$ show the $-50 \mathrm{mV}$ level. C1, The impedance profile of the $A B$ neuron in the frequency domain. The cross bar shows the resonance frequency and the maximum impedance value ( $f_{\max }=0.69 \pm 0.05 \mathrm{~Hz} ; Z_{\max }=23.5 \pm 2.3 \mathrm{M} \Omega ; n=6$ ). The resting membrane potentials for the AB trace was $-49.2 \pm 2.2 \mathrm{mV}$. The shaded region indicates $1 \mathrm{SE}$. C2, The impedance profile of the PD neuron ( $f_{\max }=0.92 \pm 0.04 ; Z_{\max }=$ $10.1 \pm 0.7 \mathrm{M} \Omega ; n=7)$. The resting membrane potentials for the PD trace was $-52.7 \pm 2.4 \mathrm{mV}$. Note that the $f_{\max }$ and $Z_{\max }$ values shown in $\mathbf{C}$ and $\mathbf{C}$ do not exactly match the peak of the mean impedance profile (solid line) attributable to the fact that the peak of the mean impedance profile is determined not only by the peak of the profiles in individual experiments but also by their slopes and amplitudes.

was similar to that observed in the biological PD neuron. Other model parameters that did not significantly affect the response of the neuron to a ZAP current injection were not changed. Because we used the model to compare with recordings done in TTX, the maximal conductances of the fast and persistent sodium currents were set to 0 . The only other modified parameters were the following maximal conductances (in $\mu \mathrm{S}): g_{\mathrm{CaT}}=$ $2.25, g_{\mathrm{CaS}}=5.4, g_{K(\mathrm{Ca})}=150$, and $g_{K d}=150$; and the time constants of $I_{\mathrm{CaT}}$ and $I_{h}$ (in $\mathrm{ms}$ ):

$$
\begin{gathered}
\tau_{m, \mathrm{CaT}}=1+9 /(1+\exp ((V+58) / 17), \\
\tau_{h, \mathrm{Ca} T}=80+10 /(1+\exp ((V+50) / 17),
\end{gathered}
$$

and

$$
\tau_{m, h}=50+200 /(1+\exp (-(V+42.2) / 8.73) .
$$

Statistical analysis. Statistical analysis was performed in SigmaStat (version 2.03; SPSS) and Origin (version 7.02; OriginLab) software packages. Error bars shown indicate SEM. In all statistical tests, data were checked for normality. Changes were considered significant if the $p$ value was below $\alpha=0.05$.

\section{Results}

Measurement of membrane resonance in pyloric pacemaker neurons

The pyloric pacemaker neurons $\mathrm{AB}$ and $\mathrm{PD}$ produce stable synchronized bursting oscillations with frequency of $\sim 1 \mathrm{~Hz}$ (Nusbaum and Beenhakker, 2002) (Fig. 1A). To examine the impedance profile of the $\mathrm{AB}$ and $\mathrm{PD}$ neurons, we superfused the preparation with saline containing $10^{-7} \mathrm{M}$ TTX to block all descending modulatory inputs and therefore stop the ongoing oscillations. We then injected a ZAP current input with frequencies sweeping from 0.2 to $3 \mathrm{~Hz}$ injected in a time interval of $60 \mathrm{~s}$ into either the $\mathrm{AB}$ or the $\mathrm{PD}$ neuron and measured the impedance $Z(f)$ as a function of frequency (see Materials and Methods). In several experiments $(n=5)$, we used additional sweeping frequencies from 0.1 to $100 \mathrm{~Hz}$ that produced an impedance profile that overlapped with those obtained in the more limited range of $0.2-3 \mathrm{~Hz}$ and did not produce any additional peaks or troughs. Additionally, changing the direction of the frequency sweep from high to low frequencies did not affect the measured impedance profile.

An example of the voltage response of these neurons to the ZAP current input is shown in Figure 1, $B 1$ for the $A B$ neuron and $B 2$ for the PD neuron. For both neurons, the impedance profile $Z(f)$ showed a characteristic peak indicating membrane resonance at a preferred frequency (henceforth referred to as $Z_{\max }$ and $f_{\max }$ ). The value of the $\mathrm{AB}$ neuron $f_{\max }$ was $0.69 \pm$ $0.05 \mathrm{~Hz}$, producing $Z_{\max }$ values of $23.5 \pm$ $2.3 \mathrm{M} \Omega(n=6)$, and those of the PD neuron were $f_{\max }=0.92 \pm 0.04 \mathrm{~Hz}$ with $Z_{\max }$ $=10.1 \pm 0.7 \mathrm{M} \Omega(n=7)$. Although, across preparations, there was some variability of the $Z_{\max }$ values for either the $\mathrm{AB}$ or the PD neuron, the resonance frequency $f_{\max }$ was relatively consistent for either neuron. Additionally, both the $f_{\max }$ and the $Z_{\max }$ values for the $\mathrm{AB}$ neuron were significantly different from those of the PD neuron (unpaired Student's $t$ test, $p=0.002$ for $f_{\max }$ and $p<0.001$ for $\left.Z_{\max }\right)$.

In the pyloric network, the two $\mathrm{PD}$ and $\mathrm{AB}$ neurons are electrically coupled; previous studies have demonstrated the dynamics of coupling between these neurons (Rabbah et al., 2005; SotoTrevino et al., 2005). These studies show that the apparent coupling between the $\mathrm{AB}$ neuron and the $\mathrm{PD}$ neuron is $<25 \%$; therefore, in case of injection of 1.5-2 nA into one PD neuron, a current with amplitude of $<0.5 \mathrm{nA}$ might be transmitted to the other PD and the $\mathrm{AB}$ neuron. Our experiments with ZAP currents with amplitudes in this range did not lead to any resonance effect (data not shown), presumably because currents in those range did not activate voltage-gated currents involved in producing resonance. We also performed the PD neuron membrane resonance measurement while the second $\mathrm{PD}$ neuron was voltage clamped at a holding potential of $-60 \mathrm{mV}$ to avoid the activation of its ionic currents. These results showed that the $f_{\max }$ of the PD neuron is not affected by voltage clamping of the other PD neuron $(n=3$; data not shown). In addition, we measured the membrane resonance property of the $\mathrm{PD}$ neuron before and after photoinactivation of the $\mathrm{AB}$ neuron. Our results showed the $f_{\max }$ of the PD neuron is independent of the $\mathrm{AB}$ neuron membrane resonance properties $(n=3)$.

The $\mathrm{AB}$ neuron is small and very sensitive to impalements. Because of the fact that our measurements with sharp electrodes require impalement of the neuron with two electrodes, we focused on the role of the membrane resonance property of the larger PD neurons, and the remainder of Results involves only data from PD neurons. 


\section{Ionic currents underlying} membrane resonance

Previous studies that have examined the underlying ionic mechanisms for membrane resonance have implicated the involvement of a variety of voltage-gated ionic currents, including the hyperpolarization-activated inward current $I_{h}$ (Puil et al., 1994; Strohmann et al., 1994), low-threshold calcium currents $I_{\mathrm{Ca}}$ (Hutcheon et al., 1994; Castro-Alamancos et al., 2007), slow non-inactivating potassium currents (Pape and Driesang, 1998; Castro-Alamancos et al., 2007), the $\mathrm{Ca}^{2+}$ activated $\mathrm{K}^{+}$current $I_{K(\mathrm{Ca})}$ (Pape and Driesang, 1998; Vigh et al., 2003), and the persistent sodium current (Wang et al., 2006). We explored the role of various ionic currents on the membrane resonance of the PD neurons using blockers.

Blocking $I_{h}$ alone (using $\mathrm{Cs}^{+}$; see Materials and Methods) resulted in an increase of the input impedance of the PD at low frequencies and greatly affected the membrane resonance (Fig. $2 A, B$ ). In two of four experiments, $\mathrm{Cs}^{+}$resulted in the disappearance of the membrane resonance and the lowest frequency $(0.15 \mathrm{~Hz})$ produced the largest impedance value; in the other two experiments, $\mathrm{Cs}^{+}$did not obliterate resonance but shifted $f_{\max }$ to a much lower frequency (range of $0.4-0.42 \mathrm{~Hz}$ ).

Blocking the calcium currents with $\mathrm{Mn}^{2+}$ had a similar effect on membrane impedance and resonance (Fig. $2 A, C$ ). However, in the presence of $\mathrm{Mn}^{2+}$, the membrane resonance property was abolished in every preparation $(n=4)$, and the peak impedance was shifted to the lowest frequency $(0.15 \mathrm{~Hz})$. Note that blocking $I_{\mathrm{Ca}}$ also results in blocking the calcium-dependent potassium current $I_{K(\mathrm{Ca})}$, and therefore these results demonstrate the effects of both currents combined. Note also that the input impedance at low frequencies is higher in $\mathrm{Mn}^{2+}$ compared with control saline. This is probably attributable to the blocking of $I_{K(\mathrm{Ca})}$, which is a large current in the PD neuron (Khorkova and Golowasch, 2007). Additionally, when both $I_{h}$ and $I_{\mathrm{Ca}}$ [and therefore $I_{K(\mathrm{Ca})}$ ] were blocked, the effect on membrane impedance was very similar to that of blocking $I_{C a}$ alone ( $n=4$; data not shown).

The fact that two distinct types of voltage-gated ionic currents were crucial for the existence of membrane resonance in the PD neurons was surprising. To understand the mechanism by which these currents could both underlie membrane resonance, we made use of a previously published model of the pyloric pacemaker neurons AB and PD (Soto-Trevino et al., 2005; Clewley et al., 2009), and, for our purposes, we only focused on the model $\mathrm{PD}$ neuron. We began by exploring the contribution of various voltage-gated ionic currents in producing membrane resonance. By manipulating or removing different maximal conductances in the model, we found that the membrane resonance in these neurons was indeed primarily determined by $I_{h}$ and $I_{\mathrm{Ca}}$. Manipulating the other ionic currents in the model did not remove the membrane resonance of the model neurons, mainly because most of the model ionic currents are activated at higher voltage thresholds. (However, some ionic currents, such as the persistent sodium current and the neuromodulator-activated inward current $I_{\text {proc }}$, had some effect on the model resonance amplitude $Z_{\max }$ or its frequency $f_{\max }$.) We therefore focused on the roles of
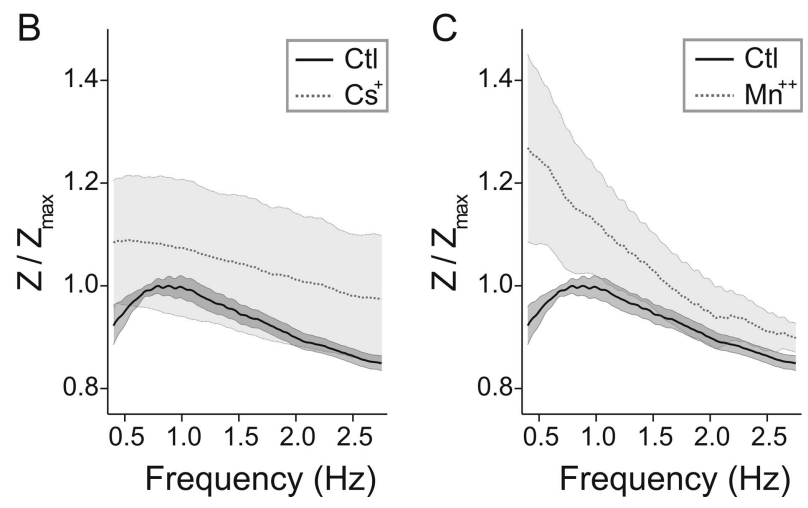

the two ionic currents $I_{h}$ and $I_{\mathrm{Ca}}$ (see Materials and Methods). Figure 3 shows the response of this model to a somatic injection of a ZAP current.

As seen in Figure $3 A$, blocking $I_{h}$ in the model changed the shape of the voltage profile in response to the ZAP current, especially at lower frequencies. In particular, the local minimum value observed in the lower envelope of the voltage profile in control disappeared after blocking $I_{h}$, resulting in a monotonically increasing lower envelope (compare dashed curves depicting the lower envelopes in Fig. 3A). We also examined the effect of decreasing or increasing of the $I_{h}$ current maximum conductances (Fig. $3 B, C$, respectively) on the impedance profile of the PD model neuron. These, respectively, had the effect of decreasing or increasing the resonance frequency by changing the impedance levels mainly at lower frequencies, as summarized in Figure $3 D$.

Blocking $I_{h}$ also affected the upper envelope of the voltage profile and shifted the voltage profile to lower voltages, resulting in the possibility that the effects on membrane impedance may be attributable to this voltage shift (Fig. $4 A$, compare bars on right). To remove this ambiguity, we injected a depolarizing DC $(0.32$ $\mathrm{nA}$ DC in addition to the ZAP current) into the model PD neuron so that the upper envelope of the voltage profile after blocking $I_{h}$ approximately matched that of the control at high frequencies (Fig. $4 B$, compare bars on the right with $A$ ). The resulting voltage profile was similar to that of blocking $I_{h}$ alone (Fig. $4 A$ ): in both cases, the membrane resonance shifted to much lower frequencies or was abolished. These simulations indicate that the role of $I_{h}$ in producing the membrane resonance in our model was primarily attributable to shaping the lower envelope of the voltage profile in response to a ZAP current input.

In contrast to the effect of blocking $I_{h}$, blocking $I_{\mathrm{Ca}}$ mainly affected the upper envelope of the voltage profile in response to a ZAP current injection, resulting in the disappearance of the local maximum value in the envelope of the voltage profile (compare dashed curves in Fig. 4C). As a result, the impedance profile after blocking $I_{\mathrm{Ca}}$ did not show a resonance peak (Fig. $4 E$ ). These results indicate that the main effect of $I_{\mathrm{Ca}}$ [in conjunction with $\left.I_{K(\mathrm{Ca})}\right]$ was to shape the upper envelope of the voltage profile.

The effect of blocking both $I_{h}$ and $I_{\mathrm{Ca}}$ in the model neuron is 

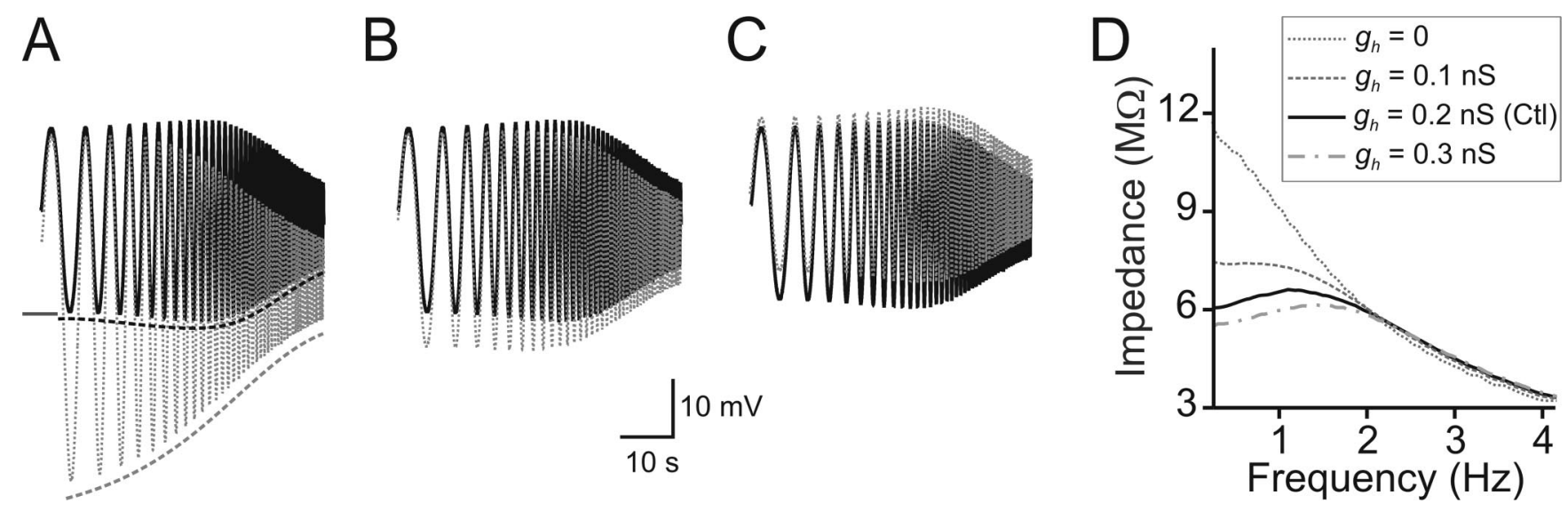

Figure 3. $I_{h}$ shapes the lower envelope of the voltage profile of the PD model neuron. All ionic currents other than $I_{\text {leak }}, I_{h}, I_{(\mathrm{Ca}}$, and $I_{K\left(C_{\mathrm{C} a}\right)}$ have been removed. $A$, The voltage response of the model PD neuron to a ZAP current injection in control (black) and after blocking $I_{h}$ (dotted gray). The dashed curves mark the lower envelope of the voltage profiles (slightly displaced for clarity), indicating the effect of $I_{h}$ in shaping the voltage response of the PD model neuron. $B$, The voltage response (dotted gray) with the maximum conductance of $l_{h}$ set to one-half of the control value of $0.2 \mathrm{n} S$ (black). $\boldsymbol{C}$, The voltage response with the maximum conductance of $I_{h}$ set to $0.3 \mathrm{nS}$ (dotted gray). The control trace is shown in black. $\boldsymbol{D}$, The impedance profile of the traces shown in $\boldsymbol{A}-\boldsymbol{C}$. Note that increasing the maximum conductance of $I_{h}$ shifts the resonance frequency to a slightly higher value. The small horizontal line in $A$ shows the $-60 \mathrm{mV}$ level in $A-C$.
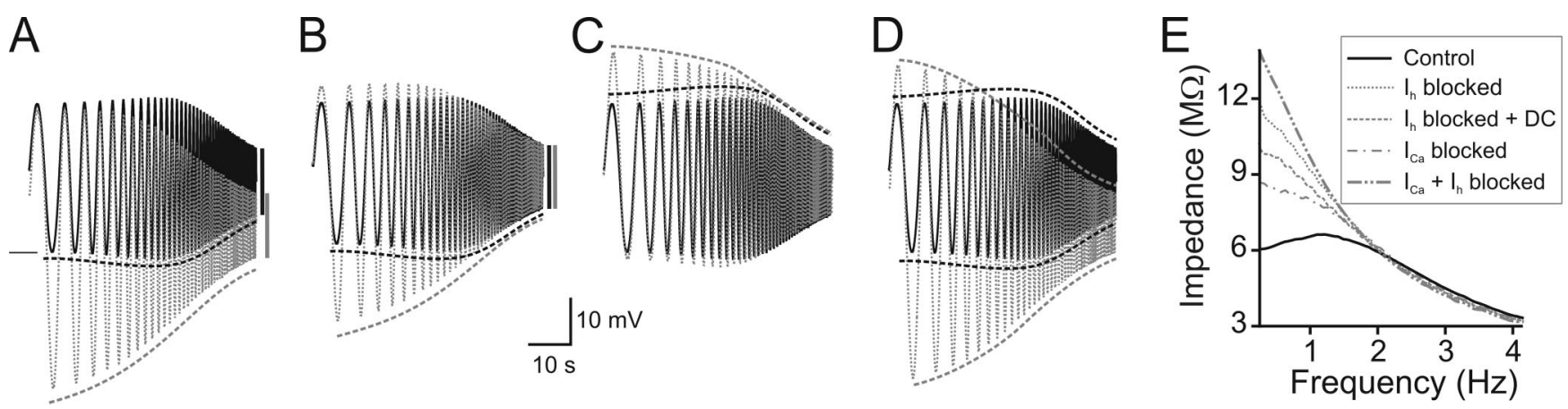

Figure 4. $I_{h}$ and $I_{C a}$, respectively, shape the lower and upper envelope of the voltage profile of the PD model neuron. $A$, Same as Figure $3 A$, showing the effect of blocking $I_{h}$ (dotted gray) on the voltage response of the model neuron. Black trace is control. Dashed curves show the lower envelope of the voltage profile, and the bars on the right indicate the voltage range at the highest frequency. $\boldsymbol{B}, A$ constant $D C$ bias current $(0.32 \mathrm{nA})$ was applied after blocking $I_{h}$ to counteract the hyperpolarized voltage attributable to blocking $l_{h}$. Even with the depolarizing bias current, the effect of blocking $I_{h}$ (dotted gray) was mainly visible in shaping the lower envelope of the voltage profile (dashed curves). Note that the upper envelope still shows a local maximum. The bars on the right indicate the voltage range at the highest frequency. $C$, The voltage response of the model PD neuron after blocking $I_{C_{a}}$ (dotted gray). Note the role of $I_{C_{a}}$ in shaping of the upper envelope of the resonance envelope indicated with the dashed curves. $\boldsymbol{D}$, Blocking both $I_{\mathrm{Ca}}$ and $I_{h}$ removes the resonance property by reshaping both upper and lower envelopes (dashed curves) of the voltage profile. $\boldsymbol{E}$, The impedance profile of the model PD neuron in control and the cases shown in $\boldsymbol{A}-\boldsymbol{D}$. Note when either the upper or lower envelopes of the voltage profile are reshaped, the resonance peak and $f_{\max }$ in the model neuron is abolished. The small horizontal line in $\boldsymbol{A}$ shows the $-60 \mathrm{mV}$ level in $\boldsymbol{A}-\boldsymbol{D}$.

shown in Figure $4 D$, indicating that blocking both currents affected both the lower and the upper envelopes of the voltage profile. Together, these modeling results indicate that $I_{h}$ and $I_{\mathrm{Ca}}$ affect the membrane impedance of the PD neurons by shaping distinct regions of the voltage profile and are therefore both necessary in producing the observed membrane resonance. We note that, in contrast to the model, in the biological PD neuron, the effect of $I_{h}$ and $I_{\mathrm{Ca}}$ in shaping the lower and upper envelopes of the voltage profiles in response to a ZAP current injection was not clearly discernible. This may be attributable to the fact that other ionic currents that contribute to this voltage profile need to be blocked to clearly show this effect.

We also examined the effect of blocking other voltage-gated ionic currents on the impedance profile of the biological PD neurons. In particular, we blocked the high-threshold potassium currents $\left[I_{K(\mathrm{Ca})}\right.$ and the delayed rectifier current $I_{K d}$ ] by bath application of $10 \mathrm{mM}$ TEA. In these experiments, however, even in the presence of TTX, injection of an external ZAP current often resulted in sporadic and irregular spike-like responses of the membrane potential in response to some cycles of the ZAP input $(n=$ 3) (Fig. 5A). These sporadic spikes occurred throughout a range of frequencies during the ZAP current injection (possibly attributable to the fact that our ZAP function only sweeps a small range of frequencies), and the measurements of input impedance versus frequency were greatly affected by the timing of the sporadic spikes and therefore the results could not be reliably compared with control. When we reduced the maximal conductance of the delayed rectifier and calcium-dependent potassium currents in our model to $20 \%$ of the original value, the model PD neurons produced sporadic spikes that were similar in shape and timing to those observed in the biological PD neuron in TEA (Fig. 5B). The model spikes were carried by calcium currents, implying the same for the biological neuron. A complete block of the potassium currents led to calcium spikes throughout the range of the ZAP current injection. We tried reducing the amplitude of the ZAP current to prevent the occurrence of these sporadic spikes (maximum membrane potential was below $-40 \mathrm{mV}$ ). Not all such sweeps showed a resonance peak. However, in sweeps in which it was possible to obtain an impedance profile, there was little difference between the resonance frequency in TEA and control conditions (as in Fig. 5C1,C2).

We used $30 \mu \mathrm{M}$ Riluzole to block the persistent sodium cur- 
rent (Wu et al., 2005) without bath application of TTX. In these experiments, we cut the descending input nerve stomatogastric nerve to remove the effect of modulatory inputs required for the production of bursting activity in the pyloric pacemaker neurons and examined the response of the PD neuron to a lowamplitude ZAP current that was subthreshold at most frequencies. Injection of such a ZAP current in the control saline produces suprathreshold activity at frequencies near the resonance frequency measured in TTX (data not shown). In addition, superfusion of Riluzole did not change the frequency range of suprathreshold activity compared with superfusion with normal saline or TTX $(n=2)$. Note that, although it is possible to reduce the amplitude of the ZAP current to move the peak membrane potential excursions to less than approximately $-40 \mathrm{mV}$ to prevent suprathreshold activity in normal saline (or Riluzole). However, such lowamplitude currents do not produce any membrane resonance in the PD neuron.

\section{Resonance and oscillation of the PD neuron}

Our results in Figure 1 indicated that the resonance frequency $f_{\max }$ of the pyloric pacemaker neurons $\mathrm{AB}$ and $\mathrm{PD}$ fall within the range of the frequencies of the ongoing pyloric rhythm. We therefore hypothesized that $f_{\max }$ of the PD neurons is correlated with the frequency of bursting oscillation of the pacemaker neurons and therefore the pyloric network frequency. It is a known fact that the oscillation frequency of these pacemaker neurons can be changed by adding a bias DC (SotoTrevino et al., 2005). We examined whether adding a bias DC also shifted the $f_{\max }$ of the PD neurons.

An example of the effect of a DC bias current injection in the PD neuron ongoing oscillation is shown in Figure $6 \mathrm{~A}$ (Ongoing), demonstrating the known fact that an increase in the DC amplitude results in faster bursting oscillations. The response of the same PD neurons (after putting the preparation in TTX) (Fig. 6A, TTX) to a ZAP current injection resulted in a shift of the largest voltage response (arrowheads), which occurs at the membrane resonance frequency $f_{\max }$. When $f_{\max }$ was plotted against the PD neuron oscillation frequency $f_{\mathrm{py}^{-}}$ loric (using the same value of injected DC: range of -1 to +1.5 $\mathrm{nA}$ ) before and application of TTX, there was a positive linear correlation between these two factors (Fig. 6B) $\left(R^{2}=0.79 \pm\right.$ $0.12 ; p<0.0001 ; n=7$ experiments; two to four data points per experiment). This correlation indicates that changes in the PD neuron $f_{\max }$ can be used as a predictive factor for changes in the ongoing oscillation frequency.

This correlation motivated us to examine whether shifting $f_{\max }$ could result in a corresponding shift in the ongoing PD neu-



C1

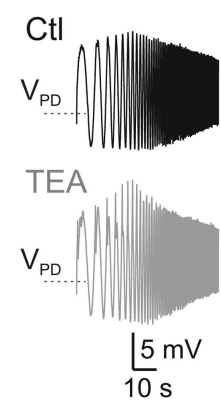

Figure 5. The effect of blocking potassium currents on the resonance profile. $\boldsymbol{A}$, An example of the effect of TEA (which blocks despite the larger impedance values, in TEA, at most frequencies. The resting membrane potentials for the TEA trace was $-50 \mathrm{mV}$.
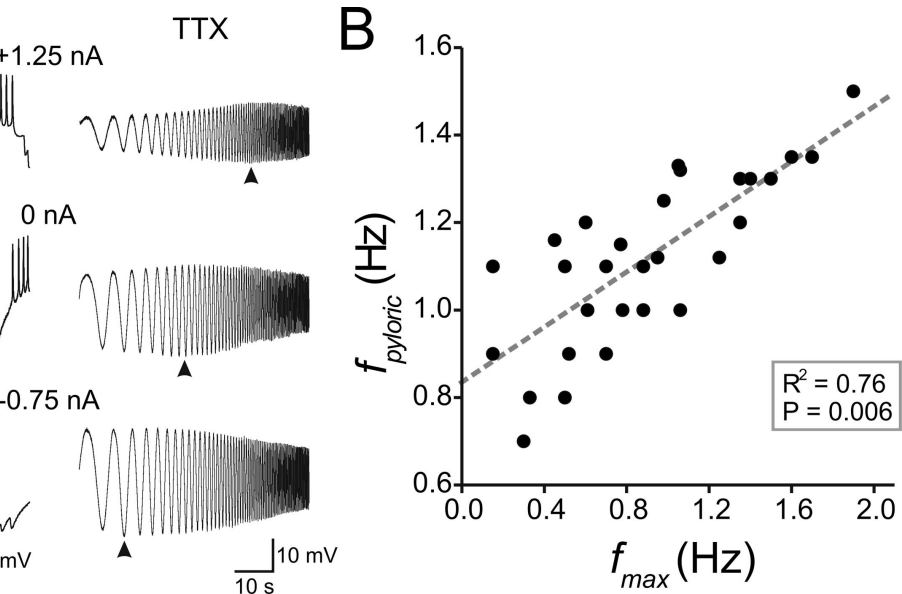

Figure 6. The resonance frequency $f_{\max }$ of the PD neuron is correlated with its ongoing oscillation frequency $f_{\text {pyloric }} \cdot \boldsymbol{A}$, Negative or positive DC injection in the PD neuron decreases or increases $f_{\text {pyloric }}$ (left). After superfusion with TTX to abolish activity, the voltage traces of the same PD neuron are shown (right) in response to the injection of a ZAP current plus the same bias current as the left column. The arrowhead shows the resonance frequency of the PD neuron. The small horizontal dashed lines show the -50 trace to the bottom, respectively. $\boldsymbol{B}$, The $f_{\text {max }}$ of the $P D$ neuron plotted versus $f_{\text {pyloric }}$ when both values are changed by injection of the same $D C$ bias currents. The dashed line shows the linear fit of the data points $\left(y=0.315 x+0.835 ; R^{2}=0.77 \pm 0.12 ; p<\right.$ $0.007 ; n=7$ experiments; $2-4$ data points per experiment).

ron oscillation frequency, thus showing the possibility of a causal relationship. To shift $f_{\max }$, we made use of the fact that the membrane resonance of the PD neurons is dependent on two distinct ionic currents, $I_{h}$ and $I_{\mathrm{Ca}}$ (Figs. 2-4). We used the dynamicclamp technique to modify the extent of either of these two currents in the PD neuron by adding (or subtracting) a dynamicclamp version of the current with a positive (or negative) maximal conductance (Bartos et al., 1999; Beenhakker et al., 2005). The implementation of an ionic current with a negative maximal conductance using dynamic clamp is equivalent to reducing the expression of that specific ionic current in the biological neuron.

We tuned the parameters of the model $I_{h}$ and $I_{\mathrm{Ca}}$ currents (used in Figs. 3, 4) so that adding either current into the PD neuron (in TTX) with dynamic clamp produced a shift in the 


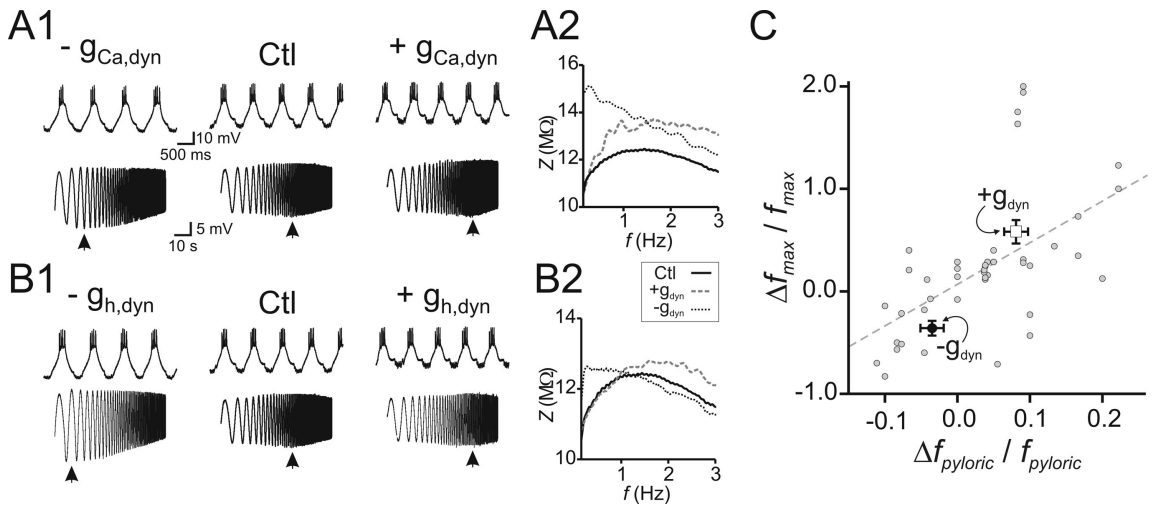

Figure 7. Alteration of the oscillation frequency $\left(f_{\text {pyloric }}\right)$ of the PD neuron by shifting its membrane resonance frequency $f_{\text {max }}$ using the dynamic-clamp technique. $\boldsymbol{A}$, Adding or subtracting $g_{\mathrm{Ca}}$ shifts both $f_{\text {max }}$ and $f_{\text {pyloric }}$ A1, Voltage traces of the PD neuron during ongoing oscillations in normal saline (top traces) and in response to a ZAP current applied in TTX (bottom traces). The left column shows the effect of subtracting a dynamic-clamp artificial $I_{\mathrm{Ca}^{\prime}}$ and the right column shows the effect of adding $I_{\mathrm{Ca}}$ (see Results). The resting membrane potentials for the traces were $-55,-48$, and $-39 \mathrm{mV}$, respectively. $\mathbf{A 2}$, Impedance profiles measured from traces in $\boldsymbol{A} \mathbf{1}$, shown as a function of frequency. $\boldsymbol{B}$, Adding or subtracting $g_{h}$ shifts both $f_{\text {max }}$ and $f_{\text {pyloric }}$. B1, As in $\boldsymbol{A 1}$, the left column shows the effect of subtracting a dynamic-clamp artificial $I_{h}$, and the right column shows the effect of adding $I_{h}$ (see Results). The resting membrane potentials for the traces were $-57,-48$, and $-41 \mathrm{mV}$, respectively. $\mathbf{B 2}$, Impedance profiles measured from traces in $\mathbf{B 1}$, shown as a function of frequency. $\boldsymbol{C}$, Normalized changes in $f_{\text {pyloric }}$ induced by changing either $I_{\mathbf{C a}}$ or $I_{h}$ are correlated to normalized changes in $f_{\text {max }}$ corresponding to the subtraction or addition of the same dynamic-clamp artificial currents. The dashed line shows a linear fit of data points $\left(R^{2}=0.57 \pm 0.088 ; p=0.001 ; n=5\right.$ experiments; up to 6 negative and 6 positive conductance values were done in each experiment). The filled circle $\left(-g_{\text {dyn }}\right)$ shows the average change obtained by the subtraction of dynamic-clamp artificial currents $\left(\Delta f_{\text {pyloric }} / f_{\text {pyloric }}=-0.035 \pm 0.016\right.$ and $\left.\Delta f_{\max } / f_{\max }=-0.36 \pm 0.072\right)$, and the open square $\left(+g_{\text {dyn }}\right)$ shows the average obtained by the addition of dynamic-clamp artificial currents $\left(\Delta f_{\text {pyloric }} / f_{\text {pyloric }}=\right.$ $0.081 \pm 0.016$ and $\left.\Delta f_{\max } / f_{\max }=0.581 \pm 0.115\right)$ for both $I_{\mathrm{Ca}}$ and $I_{h}$.

value of $f_{\max }$ in the biological neurons (see Table 1 for parameter values). We then performed a separate set of experiments to examine the effect of adding such a dynamic-clamp current on (1) the ongoing frequency $f_{\text {pyloric }}$ in control saline and (2) the PD neuron membrane resonance frequency $f_{\max }$ in TTX saline. We found that adding either $I_{h}$ or $I_{\mathrm{Ca}}$ with dynamic clamp resulted in a faster PD neuron oscillation (i.e., shift of $f_{\text {pyloric }}$ to higher values), whereas adding a negative-conductance current slowed down the oscillation (Fig. $7 A, B$ ). After putting the preparation in TTX, the same dynamic-clamp manipulation resulted in a shift of $f_{\max }$ in the same direction. To examine whether the change in $f_{\text {pyloric }}$ was correlated with the change in $f_{\max }$, we normalized the changes in either variable to the control value to reduce the variability and performed a linear regression analysis. These results showed a positive correlation between these two frequencies (Fig. $7 C)\left(R^{2}=0.57 \pm 0.088 ; p=0.001 ; n=5\right.$ experiments; two to six negative and two to six positive conductance values done in each experiment). The average shifts of $f_{\max }$ and $f_{\text {pyloric }}$ are marked by the open square (for the positive change) and filled circle (for the negative change) in Figure $7 C$.

\section{Discussion}

Neuronal bursting activity underlies the generation of network oscillations in many systems. Bursting neurons often have a wide variety of ionic currents, many of which have activation thresholds at membrane potentials well below the spike threshold. Because of the complex interaction among the nonlinear ionic currents, it is often difficult to elucidate the factors responsible for the oscillation frequency. In this study, we used the concept of membrane resonance, which has been widely used in the analysis of the subthreshold oscillations in spiking neurons, to examine the frequency setting in bursting neurons of the crab pyloric central pattern generator. The characteristics of resonance behavior of neurons capture their essential oscillatory properties within network activity near a preferred frequency (Hutcheon and Yarom, 2000). Therefore, we examined how membrane resonance properties of the neurons in the pyloric pacemaker ensemble may be correlated with the bursting cycle frequency of these neurons.

In this study, we provided three sets of results. First, we showed that the $\mathrm{PD}$ and $\mathrm{AB}$ neurons, which comprise the pyloric pacemaker ensemble, display membrane resonance and that their maximum impedance frequency $\left(f_{\max }\right)$ is within the range of the pyloric network oscillation frequency. We then focused on the PD neurons and showed that, in these neurons, membrane resonance is dependent on two distinct types of voltage gated current: the hyperpolarization-activated inward current $I_{h}$ and the calcium current $I_{\mathrm{Ca}}$. Using simulations of a computational model of the PD neuron previously developed in our laboratory, we find that $I_{h}$ and $I_{\mathrm{Ca}}$, respectively, shape the lower and upper envelopes of the voltage response to the ZAP current, thus contributing to different aspects of the voltage response that produces resonance. Finally, we showed that the membrane resonance frequency of the PD neurons is correlated with their bursting cycle frequency: factors that shift the $f_{\max }$ of the PD neuron, such as DC injection and manipulations of the conductance of $I_{h}$ and $I_{\mathrm{Ca}}$ with dynamic clamp, also change the frequency of the ongoing pyloric oscillations in the same direction.

\section{Resonance and oscillations}

Few studies have examined the role of membrane resonance in bursting neurons. One exception has been thalamic neurons. The thalamus is composed of a conglomerate of nuclei involving neurons with significantly different properties and is believed by many researchers to be the pacemaker network for spindle rhythms during sleep (Huguenard, 1998). Neurons of the medial geniculate nucleus, for example, demonstrate a resonance peak at $1 \mathrm{~Hz}$ that is influenced by a T-type Ca current (Tennigkeit et al., 1997). Many oscillatory neurons have been shown to have membrane resonance properties that are correlated with the network oscillatory activity. Inferior olive neurons show an impedance peak between 3 and $10 \mathrm{~Hz}$, which corresponds to the frequency of the spontaneous oscillations in these neurons (Lampl and Yarom, 1997). Additionally, it has been shown that bursting activity and resonance frequency in cerebellar granule cells occur within the theta-frequency range (D'Angelo et al., 2001). Guinea pig frontal cortex neurons show membrane resonance in the range of 3-15 Hz based on the level of depolarization. These neurons generate subthreshold oscillations at these frequencies and preferentially respond to inputs arriving at similar frequencies (Gutfreund et al., 1995). Mesencephalic trigeminal neurons also show rhythmic bursting activity as well as membrane resonance (Enomoto et al., 2007). Moreover, several studies have claimed that the basis of the theta rhythm in the hippocampal formation can be attributed to the membrane resonance frequency of CA1 pyramidal neurons and the entorhinal cortical 
stellate cells (Hu et al., 2002; Peters et al., 2005; Wang et al., 2006; Giocomo et al., 2007).

\section{Ionic currents underlying resonance}

It has been proposed that any ionic current that oppose changes in the membrane voltage and has a slow time constant can produce resonance (Hutcheon and Yarom, 2000). A variety of such currents: $I_{h}$, slow non-inactivating potassium currents $I_{M}$, and the calcium-activated potassium current $I_{K(\mathrm{Ca})}$ have been shown to contribute to membrane resonance in different neurons (Puil et al., 1994; Pape and Driesang, 1998; Hu et al., 2002; Vigh et al., 2003; Wang et al., 2006; Castro-Alamancos et al., 2007). Additionally, the persistent sodium current $I_{\mathrm{NaP}}$ and low-threshold calcium current $I_{\mathrm{Ca}}$, which act to amplify subthreshold signals, are known to contribute to membrane resonance (D'Angelo et al., 2001; Hu et al., 2002; Castro-Alamancos et al., 2007; Enomoto et al., 2007). Many of these ionic currents $\left(I_{h}, I_{\mathrm{NaP}}, I_{\mathrm{Ca}}\right.$, and $\left.I_{M}\right)$ also contribute to pacemaker activities (Del Negro et al., 2005; Forti et al., 2006; Peck et al., 2006; Tazerart et al., 2008).

In the PD neuron, the main currents contributing to membrane resonance appear to be $I_{h}, I_{\mathrm{Ca}}$, and $I_{K(\mathrm{Ca})}$, although we have not completely ruled out the indirect influence of other voltagegated currents. Blocking potassium currents with TEA or reducing $I_{K(\mathrm{Ca})}$ in the model led to sporadic (putative) calcium spikes that interfered with impedance measurements. Blocking calcium currents led to a dramatic increase in input impedance (Fig. $2 \mathrm{~A}, \mathrm{C}$ ) possibly because of the blockage of $I_{K(\mathrm{Ca})}$, which is large in these neurons (Khorkova and Golowasch, 2007). Because there is no way for blocking the calcium currents without also removing $I_{K(\mathrm{Ca})}$, it is not easy to tease apart the roles of $I_{\mathrm{Ca}}$ and $I_{K(\mathrm{Ca})}$ in producing resonance. Theoretical studies have shown that autorhythmicity can be produced by any mechanism that produces membrane resonance at a preferred frequency (Holden, 1980). As such, low-threshold calcium currents may be an essential contributor to the production of oscillations or determining the oscillation frequency in the pyloric pacemaker neurons. Few studies have explored the dynamics of calcium currents in stomatogastric neurons (Hurley and Graubard, 1998; Johnson et al., 2003), and their role in rhythmogenesis in this network remains to be explored.

\section{Resonance and network effects}

Some modeling studies have indicated that networks of neurons can demonstrate resonance properties that depend on short-term plasticity of synaptic inputs (Houweling et al., 2002; Maex and De Schutter, 2005). However, in experimental studies, alteration of network frequency by means of manipulation of its synaptic properties has not been reported. It has been shown that shortterm plasticity in synapses can produce a maximal synaptic response at a preferred frequency, a property that could be called synaptic resonance (Izhikevich et al., 2003). In a previous study, we examined the interaction between membrane and synaptic resonance (Drover et al., 2007). We showed that a neuron with resonance properties that receives input from a synapse that also shows resonance would have a maximal response at a frequency that lies between the two resonance frequencies. In the current study, synaptic effects were absent in the measurements of the membrane resonance properties because these measurements were done in TTX, which blocks all activity, including activity of neurons presynaptic to the PD neuron. As such, the role of synaptic resonance properties on the oscillatory activity of CPG networks in general and the pyloric network in particular remains to be explored.
A correlation between the resonance frequency of the pacemaker neurons and the bursting oscillation frequency suggests that membrane resonance could be an important factor in setting the frequency of pacemaker-driven networks. However, in large multilayer networks, the influence of the membrane resonance of the pacemaker neurons could be quite different. Modeling simulations of such multilayer networks have shown that the entrainment frequency window by pacemaker neurons decreases exponentially with depth, and therefore only small networks can show frequency locking to the pacemaker neurons (Kori and Mikhailov, 2004). However, studies in other systems have also indicated that membrane resonance can be related to the network oscillation frequency. The onset of whisking in newborn and young rats, for example, has been shown to be coincident with the expression of $I_{h}$ and appearance of subthreshold membrane resonance in rat vibrissa motoneurons (Nguyen et al., 2004). Experiments on layer $\mathrm{V}$ pyramidal neurons of the cortex have also underlined the bandpass filtering property of these neurons especially at the dendritic level (Ulrich, 2002). Interestingly, a modeling study shows that networks that include different neuronal subtypes, some with resonance properties and others with integrating properties, can produce network stability or flexible responsiveness to external stimuli, depending on the structure of the input spike trains (Muresan and Savin, 2007).

Although most studies have focused on the role of membrane resonance in the selectivity of response to inputs at certain frequencies, our findings underline the role of membrane resonance in determining the oscillation frequency of the whole network. In particular, our findings underline the role of the resonance frequency of the pacemaker neurons in determining the oscillation frequency of the full pyloric CPG. Pyloric oscillations directly control the filtering of food by the pylorus (Harris-Warrick et al., 1992) but also indirectly influence the chewing of food by the gastric mill (Bartos et al., 1999). As such, factors that influence the pyloric CPG frequency can affect a combination of feeding behaviors in the animal. The pyloric pacemaker neurons also receive synaptic input from follower pyloric neurons at the same frequency. It is therefore possible that, in these pacemaker neurons, the membrane resonance property acts both to set the intrinsic bursting frequency and to act as an enhancing mechanism for the synaptic inputs that arrive at this frequency. We also predict that extrinsic and neuromodulatory factors that change $I_{h}$ or $I_{\text {Ca }}$ (Johnson et al., 2003; Peck et al., 2006) or affect the way these currents interact with other membrane or synaptic properties may change the membrane resonance frequency or the impedance profile and consequently change the oscillation frequency of these neurons.

\section{References}

Art JJ, Crawford AC, Fettiplace R (1986) Electrical resonance and membrane currents in turtle cochlear hair cells. Hear Res 22:31-36.

Bartos M, Manor Y, Nadim F, Marder E, Nusbaum MP (1999) Coordination of fast and slow rhythmic neuronal circuits. J Neurosci 19:6650-6660.

Beenhakker MP, DeLong ND, Saideman SR, Nadim F, Nusbaum MP (2005) Proprioceptor regulation of motor circuit activity by presynaptic inhibition of a modulatory projection neuron. J Neurosci 25:8794-8806.

Bellingham MC (1998) Driving respiration: the respiratory central pattern generator. Clin Exp Pharmacol Physiol 25:847-856.

Brunel N, Hakim V, Richardson MJ (2003) Firing-rate resonance in a generalized integrate-and-fire neuron with subthreshold resonance. Phys Rev E Stat Nonlin Soft Matter Phys 67:051916.

Castro-Alamancos MA, Rigas P, Tawara-Hirata Y (2007) Resonance (approximately $10 \mathrm{~Hz}$ ) of excitatory networks in motor cortex: effects of voltage-dependent ion channel blockers. J Physiol 578:173-191. 
Clewley R, Soto-Treviño C, Nadim F (2009) Dominant ionic mechanisms explored in spiking and bursting using local low-dimensional reductions of a biophysically realistic model neuron. J Comput Neurosci 26:75-90.

D’Angelo E, Nieus T, Maffei A, Armano S, Rossi P, Taglietti V, Fontana A, Naldi G (2001) Theta-frequency bursting and resonance in cerebellar granule cells: experimental evidence and modeling of a slow $\mathrm{K}^{+}$dependent mechanism. J Neurosci 21:759-770.

Del Negro CA, Morgado-Valle C, Hayes JA, Mackay DD, Pace RW, Crowder EA, Feldman JL (2005) Sodium and calcium current-mediated pacemaker neurons and respiratory rhythm generation. J Neurosci 25:446-453.

Drover JD, Tohidi V, Bose A, Nadim F (2007) Combining synaptic and cellular resonance in a feed-forward neuronal network. Neurocomputing 70:2041-2045.

Engel TA, Schimansky-Geier L, Herz AV, Schreiber S, Erchova I (2008) Subthreshold membrane-potential resonances shape spike-train patterns in the entorhinal cortex. J Neurophysiol 100:1576-1589.

Enomoto A, Han JM, Hsiao CF, Chandler SH (2007) Sodium currents in mesencephalic trigeminal neurons from Nav1.6 null mice. J Neurophysiol 98:710-719.

Forti L, Cesana E, Mapelli J, D’Angelo E (2006) Ionic mechanisms of autorhythmic firing in rat cerebellar Golgi cells. J Physiol 574:711-729.

Gimbarzevsky B, Miura RM, Puil E (1984) Impedance profiles of peripheral and central neurons. Can J Physiol Pharmacol 62:460-462.

Giocomo LM, Zilli EA, Fransén E, Hasselmo ME (2007) Temporal frequency of subthreshold oscillations scales with entorhinal grid cell field spacing. Science 315:1719-1722.

Goaillard JM, Marder E (2006) Dynamic clamp analyses of cardiac, endocrine, and neural function. Physiology 21:197-207.

Golowasch J, Marder E (1992) Proctolin activates an inward current whose voltage dependence is modified by extracellular $\mathrm{Ca}^{2+}$. J Neurosci 12:810-817.

Gutfreund Y, Yarom Y, Segev I (1995) Subthreshold oscillations and resonant frequency in guinea-pig cortical neurons: physiology and modelling. J Physiol 483:621-640.

Harris-Warrick RM, Marder E, Selverston AI, Moulins M (1992) Dynamic biological networks: the stomatogastric nervous system. Cambridge, MA: MIT.

Holden AV (1980) Autorhythmicity and entrainment in excitable membranes. Biol Cybern 38:1-8.

Hooper SL, Marder E (1984) Modulation of a central pattern generator by two neuropeptides, proctolin and FMRFamide. Brain Res 305:186-191.

Houweling AR, Bazhenov M, Timofeev I, Grenier F, Steriade M, Sejnowski TJ (2002) Frequency-selective augmenting responses by short-term synaptic depression in cat neocortex. J Physiol 542:599-617.

Hu H, Vervaeke K, Storm JF (2002) Two forms of electrical resonance at theta frequencies, generated by $\mathrm{M}$-current, h-current and persistent $\mathrm{Na}+$ current in rat hippocampal pyramidal cells. J Physiol 545:783-805.

Huguenard JR (1998) Anatomical and physiological considerations in thalamic rhythm generation. J Sleep Res 7 [Suppl 1]:24-29.

Hurley LM, Graubard K (1998) Pharmacologically and functionally distinct calcium currents of stomatogastric neurons. J Neurophysiol 79:2070-2081.

Hutcheon B, Yarom Y (2000) Resonance, oscillation and the intrinsic frequency preferences of neurons. Trends Neurosci 23:216-222.

Hutcheon B, Miura RM, Yarom Y, Puil E (1994) Low-threshold calcium current and resonance in thalamic neurons: a model of frequency preference. J Neurophysiol 71:583-594.

Izhikevich EM, Desai NS, Walcott EC, Hoppensteadt FC (2003) Bursts as a unit of neural information: selective communication via resonance. Trends Neurosci 26:161-167.

Johnson BR, Kloppenburg P, Harris-Warrick RM (2003) Dopamine modulation of calcium currents in pyloric neurons of the lobster stomatogastric ganglion. J Neurophysiol 90:631-643.

Khorkova O, Golowasch J (2007) Neuromodulators, not activity, control coordinated expression of ionic currents. J Neurosci 27:8709-8718.

Kori H, Mikhailov AS (2004) Entrainment of randomly coupled oscillator networks by a pacemaker. Phys Rev Lett 93:254101.

Lampl I, Yarom Y (1997) Subthreshold oscillations and resonant behavior: two manifestations of the same mechanism. Neuroscience 78:325-341.
Leung LS, Yu HW (1998) Theta-frequency resonance in hippocampal CA1 neurons in vitro demonstrated by sinusoidal current injection. J Neurophysiol 79:1592-1596.

Llinás R, Yarom Y (1986) Oscillatory properties of guinea-pig inferior olivary neurones and their pharmacological modulation: an in vitro study. J Physiol 376:163-182.

Llinás RR (1988) The intrinsic electrophysiological properties of mammalian neurons: insights into central nervous system function. Science 242:1654-1664.

Maex R, De Schutter E (2005) Oscillations in the cerebellar cortex: a prediction of their frequency bands. Prog Brain Res 148:181-188.

Miller JP, Selverston A (1979) Rapid killing of single neurons by irradiation of intracellularly injected dye. Science 206:702-704.

Moulins M, Cournil I (1982) All-or-none control of the bursting properties of the pacemaker neurons of the lobster pyloric pattern generator. J Neurobiol 13:447-458.

Muresan RC, Savin C (2007) Resonance or integration? Self-sustained dynamics and excitability of neural microcircuits. J Neurophysio 97:1911-1930.

Nguyen QT, Wessel R, Kleinfeld D (2004) Developmental regulation of active and passive membrane properties in rat vibrissa motoneurones. J Physiol 556:203-219.

Nusbaum MP, Beenhakker MP (2002) A small-systems approach to motor pattern generation. Nature 417:343-350.

Pape HC, Driesang RB (1998) Ionic mechanisms of intrinsic oscillations in neurons of the basolateral amygdaloid complex. J Neurophysiol 79:217-226.

Peck JH, Gaier E, Stevens E, Repicky S, Harris-Warrick RM (2006) Amine modulation of Ih in a small neural network. J Neurophysiol 96:2931-2940.

Peters HC, Hu H, Pongs O, Storm JF, Isbrandt D (2005) Conditional transgenic suppression of $\mathrm{M}$ channels in mouse brain reveals functions in neuronal excitability, resonance and behavior. Nat Neurosci 8:51-60.

Puil E, Gimbarzevsky B, Miura RM (1986) Quantification of membrane properties of trigeminal root ganglion neurons in guinea pigs. J Neurophysiol 55:995-1016.

Puil E, Meiri H, Yarom Y (1994) Resonant behavior and frequency preferences of thalamic neurons. J Neurophysiol 71:575-582.

Rabbah P, Golowasch J, Nadim F (2005) Effect of electrical coupling on ionic current and synaptic potential measurements. J Neurophysiol 94:519-530.

Richardson MJ, Brunel N, Hakim V (2003) From subthreshold to firingrate resonance. J Neurophysiol 89:2538-2554.

Selverston AI, Russell DF, Miller JP (1976) The stomatogastric nervous system: structure and function of a small neural network. Prog Neurobiol 7:215-290.

Soto-Treviño C, Rabbah P, Marder E, Nadim F (2005) Computational model of electrically coupled, intrinsically distinct pacemaker neurons. J Neurophysiol 94:590-604.

Ströhmann B, Schwarz DW, Puil E (1994) Subthreshold frequency selectivity in avian auditory thalamus. J Neurophysiol 71:1361-1372.

Tazerart S, Vinay L, Brocard F (2008) The persistent sodium current generates pacemaker activities in the central pattern generator for locomotion and regulates the locomotor rhythm. J Neurosci 28:8577-8589.

Tennigkeit F, Ries CR, Schwarz DW, Puil E (1997) Isoflurane attenuates resonant responses of auditory thalamic neurons. J Neurophysiol 78:591-596.

Ulrich D (2002) Dendritic resonance in rat neocortical pyramidal cells J Neurophysiol 87:2753-2759.

Vigh J, Solessio E, Morgans CW, Lasater EM (2003) Ionic mechanisms mediating oscillatory membrane potentials in wide-field retinal amacrine cells. J Neurophysiol 90:431-443.

Wang WT, Wan YH, Zhu JL, Lei GS, Wang YY, Zhang P, Hu SJ (2006) Theta-frequency membrane resonance and its ionic mechanisms in rat subicular pyramidal neurons. Neuroscience 140:45-55.

Wu N, Enomoto A, Tanaka S, Hsiao CF, Nykamp DQ, Izhikevich E, Chandler SH (2005) Persistent sodium currents in mesencephalic v neurons participate in burst generation and control of membrane excitability. J Neurophysiol 93:2710-2722. 